\title{
Breast Cancer Histopathological Image Classification using Deep Convolutional Neural Network
}

\author{
Vishal Mellahalli Siddegowda \\ Department of computing and \\ informatics \\ Bournemouth University \\ Bournemouth, GB \\ vishalsiddegowda96@gmail.com
}

\begin{abstract}
Deep learning has come up with the intense class of models which have potential applications in the field of image classification, video recognition, object recognition, natural language Processing and speech recognition. Mainly, Deep convolutional Neural Network is one of the deep learning models that is used for image classification, that extracts the feature from the images and use these extracted features to classify images (2D or 3D images). In this paper, DCNN is used to classify mammogram images obtained from medical imaging process to detect the benign and malignant cells. The outcome of the study is to bring out the idea behind computing techniques incorporated with medical diagnostics, helping medical professional to take advantage of computer aided diagnostics, ultimately improving the time spent by pathologist to inspect the stained tissues in-turn increasing the survival rates.
\end{abstract}

Keywords-breast cancer, mammogram, deep learning, transfer learning, data augmentation

\section{INTRODUCTION}

The global trend in reported breast cancer mortalities and cases has increased significantly in recent years. [6] states that " 2.1 million new cases and 627,000 deaths were reported in 2018 as against 1.7 million new cases and 521,900 deaths in 2012". WHO declares that breast cancer is one of the most fatal cancerous disease in women both in developed and less developed countries leading to unimaginable death rates in women population. It has been estimated that 508000 women died due to breast cancer worldwide during the year 2011. Many researchers suggest that the low survival rate in breast cancer can mainly be explained by lack of early-stage cancer detection program leading to final stage diseases, and as well as inadequate diagnostic equipment's in most of developing countries.

The fundamental idea behind deep learning techniques is to train the computer-based machine learning models to recognize/detect pattern in the training set and make impressive prediction on the future data. Among many available deep learning models, CNN's have proven to be the efficient in image recognition, classification, and segmentation. [6] mentions that the unique ability of CNN is to learn the inherent feature/pattern in the input data though lacking feature planning. Basically, CNN's consist of many phases of learnable filter convolved with the data (image, video, or speech) followed by multilayer perceptron. The convolutional layer and the multilayer perceptron are accommodated by non-linear activation function such as sigmoid function, hyperbolic tangent function, rectified liner unit (ReLU) or leaky rectified liner unit (leaky ReLU), finally obtaining the output.

\section{II.JUSTIFICATION}

Deep convolutional neural network has come out to be one of the best images classifying algorithm in the field of computer vision and intervention of these artificial intelligence technique into medical prognosis can result in immense improvement in early detection of breast carcinoma.

Mammogram method has shown a favorable way of examining the cancerous cells that helps for early detection. [1] states that histopathology is a field of study that aims to learn about cancer cells which is brought up by medical imaging(mammogram). In histopathology, chemicals hematoxylin and eosin are used to stain the tissue under test to differentiate between areas of tissue. Pathologists are the medical professional who study on these stained tissues to understand the cancerous and noncancerous cells. Since there exists clinical audits to ensure minimum diagnostics error, many pathologists needs to provide their inference on the cancer cells imaging which makes authentication process expensive and time consuming. And difference in opposing conclusion between pathologists makes the task impossible. Thus, computer aided diagnostics comes into picture that can be a viable solution for early detection in breast cancer which can be achieved in minimal time.

Deep learning architecture plays a vital role in image classification, especially convolutional neural network has shown promising outcome in many research and experiments conducted on cancer cells detection. Yet, many researchers are trying to intensify the outcome and it is open for improvisation in the coming future.

\section{III.HYPOTHESIS TO BE TESTED}

Hypothesis includes many key areas to be tested in breast cancer detection using deep convolutional neural network as follows

- Does the chosen convolutional neural network architecture performs well compared to the other machine learning algorithms such as SVM, Random forest, logistic regression and so on?

- Does size of the dataset have impact on performance of the algorithm? 
- Is transfer learning a necessary technique to incorporate whilst training a small dataset?

- Which deep learning model would result in classifying cancerous cells with highest accuracy?

\section{IV.METHODOOLOGY}

\section{A. Artificial Neural Network}

Artificial Neural Networks (ANN) was built on the idea which originated from the biological nervous system specifically human brain and these systems behave as a basic building blocks in any deep neural networks which vaguely mimics human neuronal system.

[12] expresses that "ANN consists of high number of interconnected computational nodes referred as neurons which work entwine in a distributed fashion to collectively learn from the input in order to optimize its final output". Typically, ANN incorporates three layers such as input, hidden, and output layers. Each circular structure represents neurons, to which input is fed and output is extracted.

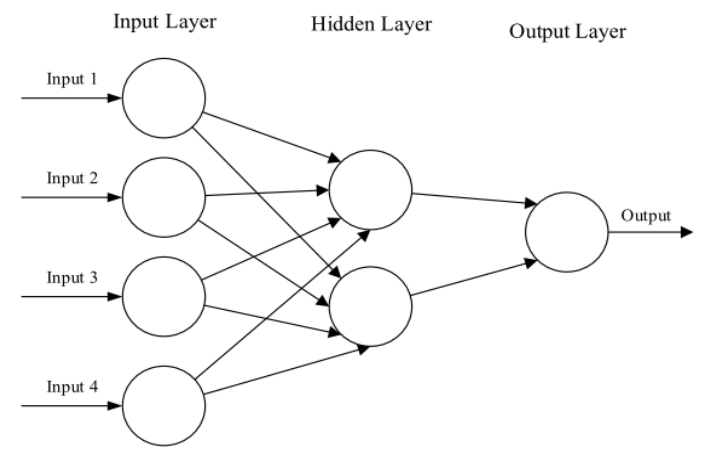

Fig 1: Typical Artificial Neural Network [12]

\section{B. Basic Convolutional Neural Network \\ Architecture:}

Convolutional neural network (CNN) is basically analogous to artificial neural network with added convolving feature resulting in $\mathrm{CNN}$ and known as ConvNets. CNN is so named as it consists of convolutional layer included in its architecture. [6] justifies that convolution neural network includes multiple stages of learnable filters that are multiplied with the inputs (images, audio, or video). Convolutional layers are designed to learns the features of the input images in each step using different filter size (weights and biases) at different time.

A deep convolutional Neural Network is trained by inputting the images into the first layers and allowing it to identify the hidden features (horizontal and vertical edge if it is an image) and finally generating the output using activation function (ReLu/sigmoid/Tan). Once the output is obtained, error is calculated using loss function, and this error is backpropagated to find out the lowest optima. The main goal on $\mathrm{CNN}$ is to fine tune the parameters to reach the smallest error possible. Training a CNN is multi-step

process that contains multiple layers through which the images are being classified.

There are three important layers in convolutional neural network: convolution layer, pooling layer, and fully connected layer. And each of these layers are stacked one after another several times depending on the architecture. A general ConvNets with single stage is shown below.

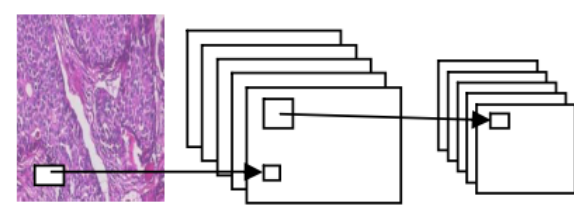

Input Image Convolution + Pooling Fully Connected
Non Linearity

Fig 2: Example of typical CNN architecture classifying cancer image [5]

The first and the most important layer in $\mathrm{CNN}$ is convolution kernel as shown in figure 2 (filters with weights), which is characterized to perform dot product on input image and weights which results in identifying important features of input image. Let us say input image is of size $512 * 512$ which is $3 \mathrm{D}$ image (RGB), for such image filters could be of size $(3 * 3$ or $5 * 5$ or $8 * 8)$ and each neuron from previous layers (i.e., image) are connected to this layer (i.e., filter).

Further, pooling layers can be stacked in between two convolution layers and its goal is to diminish the spatial dimension of the input image whilst retaining all the important features. Therefore, pooling helps in reducing the quantity of parameters and calculation required by the neural network which in turn avoids overfitting. Pooling can be performed with two different criteria i.e., Max pooling and average pooling, but max pooling is preferred in the recent times because of its impact on output performance. Once the pooling process is completed, all the data in the matrix form are flattened before feeding them into fully connected layer.

Finishing layer would be Fully Connected layer, [5] briefs that all the flattened neuronal nodes from the present layers are connected to every other node of the succeeding layer. And their activation value can be calculated by matrix multiplication followed by addition of bias offset. The last fully connected layer has the net output, and the final classification task is performed in these layers.

\section{Common Evaluation Metrics}

Following metrics can be used to check the classification performance:

1. Confusion Matrix: A confusion matrix consists of information regarding the actual and predicted classes performed by classifying systems. Given that it consists of $n$ different classes, the size of confusion matrix would be $n * n$. 


\section{Predicted class}

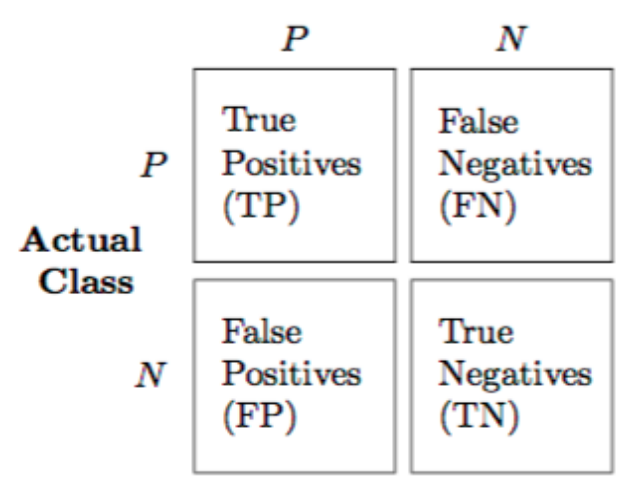

- True Positive (TP): These are the cases where prediction is positive, which means system has successfully identified the cancerous cell and its true.

- True Negative (TN): These are the cases where prediction is negative, which means system has classified the image as noncancerous and its true.

- False Positive (FP): These are the cases where prediction is positive, which means that the system has recognized the image as cancerous and its false.

- False Negative (FN): These are the cases where prediction is negative, which means that system has identified the image as noncancerous and its false.

i) Classification Accuracy: It is the ratio of correctly predicted cancer images to the total prediction made.

$$
\text { Accuracy }=(T P+T N) /(T P+T N+F P+F N)
$$

ii) Precision: It is the ability of classification system to correctly classify the cancerous and non-cancerous images in terms of true positive (TP) and false positive (FP).

$$
\text { Precision }=(\mathrm{TN}) /(\mathrm{FP}+\mathrm{TN})
$$

iii) Recall: It measure the performance of classifying system in terms true positive (TP) and false negative (FN).

$$
\text { Recall }=(T P) /(T P+F N)
$$

2. Receiver Operating Characteristic (ROC) Curve and Area Under ROC Curve (AUC): ROC curve is a graphical plot that emphasize on the discrimination capability of the system at various discrimination threshold. Higher AUC depicts that the model has higher performance

\section{V.DISCUSSION}

The following discussions would give an overview of how researchers have formulated their methodology to achieve their outcome, respectively. Deep learning has played a significant role in the field of medical science and shows a concrete evidence in its performance towards identifying the abnormal cells from normal cells. [4] and [6] has incorporated deep convolutional neural network technique to classify the breast cancer cells but the keynote in their research is that they have used original/raw size images in the training set to perform classification. However, other researchers namely [3] has used patch of images created from patch generation to train their algorithms. Thus, the researchers have integrated different techniques with DCNN to enhance their output performance.

[1] In his research has shown that using data augmentation technique would result in creating more sample sets which avoids overfitting and boosts the performance of the classifier. [2] have come up with research where they have used two different deep learning model such as VGG16 and ResNet50 to classify normal/abnormal cell and the results were quite promising. Thus, different opinions from different researchers have left the hypothesis in perplexed state and needs to be tested universally.

Ensuing discussion explains the results of tested hypothesis, Deep convolutional neural network has always shown optimistic (table 1) results in field of computer vision (image classification, speech recognition, object detection etc.). Deep convolution neural networks have better performance measure compared to traditional machine learning algorithms. [11] propose that high performance of neural networks is because neural nets roughly imitate the brain activity, and several neural network hidden layers are stacked one after another like traditional brain model. However, there is no such empirical evidence for deep learning.

Smaller training dataset will of course have impact on output performance which leads to overfitting, thus the technique called data augmentation comes into picture that outputs mirroring images - horizontal and vertical flips, rotated images, random cropped images and color shifting which ultimately enhances the size and quality of the training datasets. [9] justifies that the redundant data can be removed by data augmentation technique and it also helps in reducing the probability of overfitting.

Transfer learning is used in major instances in deep learning because of its ability to train the deep neural network with comparatively little data as suggested by [2]. Many a times these training may take couple of weeks and might consume high graphic processing. In fact, someone else would have gone through many complex processes to reach the final point, however one can download all the necessary neural network architecture and use that as one's own work with their initialization procedure. 


\begin{tabular}{|c|c|c|}
\hline Author & Architecture & $\begin{array}{l}\text { Accuracy } \\
\text { Achieved }\end{array}$ \\
\hline $\begin{array}{l}\text { Ankit titoriya et. } \\
\text { al. (2019) }\end{array}$ & AlexNet & $\begin{array}{l}\text { Accuracy that } \\
\text { ranges between } \\
93.8 \% \text { and } \\
95.7 \% \text {. }\end{array}$ \\
\hline $\begin{array}{l}\text { Angara et. al. } \\
(2019)\end{array}$ & Basic CNN & $\begin{array}{l}\text { Accuracy with } \\
\text { an average } \\
\text { image level } \\
\text { accuracy of } \\
\text { about } 91 \% \text { and } \\
\text { patient scores } \\
\text { as high as } 95 \% .\end{array}$ \\
\hline $\begin{array}{l}\text { Ismail and } \\
\text { Sovuthy (2019) }\end{array}$ & $\begin{array}{l}\text { VGG16, } \\
\text { ResNet50 }\end{array}$ & $\begin{array}{l}\text { VGG-16- 94\% } \\
\text { ResNet50 } \\
91.7 \%\end{array}$ \\
\hline
\end{tabular}

Table 1: Comparing empirical results of different experiments

\section{VI.CONCLUSION}

In summary, this paper deals with detailed structure of classifying the hematoxylin and eosin-stained cancer images and table 1 depicts the accuracy comparison of the state-of-the-art architecture. Paper also emphasizes on the key aspects on deep learning such as deep convolutional neural network outperforms traditional machine learning algorithms, data augmentation plays a crucial role in reducing the effect of overfitting on the algorithm, transfer learning is an interesting technique that helps the deep learning enthusiasts to incorporate pre-trained model to use it on their application without having to build model from scratch.

Basically, all the deep convolutional neural networks have shown a significant performance in classifying the stained tissues of breast cancer but few neural network architectures such as AlexNet, VGG16 and ResNet50 have stood out with their high efficiency. Yet there is abundance amount of room for significant improvement over course of time.

\section{REFERENCES}

[1] A. Roy, "Deep Convolutional Neural Networks for Breast Cancer Detection," 2019 IEEE 10th Annual Ubiquitous Computing, Electronics \& Mobile Communication Conference (UEMCON), New York City, NY, USA, 2019, pp. 0169-0171, doi: 10.1109/UEMCON47517.2019.8993023.

[2] N. S. Ismail and C. Sovuthy, "Breast Cancer Detection Based on Deep Learning Technique," 2019 International UNIMAS STEM 12th Engineering Conference (EnCon), Kuching, Malaysia, 2019, pp. 89-92, doi: 10.1109/EnCon.2019.8861256.

[3] S. Angara, M. Robinson, and P. Guillén-Rondon, "Convolutional Neural Networks for Breast Cancer Histopathological Image Classification," 2018 4th International Conference on Big Data and Information
Analytics (BigDIA), Houston, TX, USA, 2018, pp. 1-6, doi: 10.1109/BigDIA.2018.8632800.

[4] T. Kausar, M. Wang and M. S. S. Malik, "Cancer Detection in Breast Histopathology with Convolution Neural Network Based Approach," 2019 IEEE/ACS 16th International Conference on Computer Systems and Applications (AICCSA), Abu Dhabi, United Arab Emirates, 2019, pp. 1-5, doi: 10.1109/AICCSA47632.2019.9035244.

[5] K. Kumar and A. C. S. Rao, "Breast cancer classification of image using convolutional neural network," 2018 4th International Conference on Recent Advances in Information Technology (RAIT), Dhanbad, 2018, pp. 1-6, doi: 10.1109/RAIT.2018.8389034.

[6] P. Yamlome, A. D. Akwaboah, A. Marz and M. Deo, "Convolutional Neural Network Based Breast Cancer Histopathology Image Classification," 2020 42nd Annual International Conference of the IEEE Engineering in Medicine \& Biology Society (EMBC), Montreal, QC, Canada, 2020, pp. 1144-1147, doi: 10.1109/EMBC44109.2020.9176594.

[7] K. Liu, G. Kang, N. Zhang, and B. Hou, "Breast Cancer Classification Based on Fully-Connected Layer First Convolutional Neural Networks," in IEEE Access, vol. 6, pp. 23722-23732, 2018, doi: 10.1109/ACCESS.2018.2817593.

[8] C. Muramatsu, Y. Hiramatsu, H. Fujita and H. Kobayashi, "Mass detection on automated breast ultrasound volume scans using convolutional neural network," 2018 International Workshop on Advanced Image Technology (IWAIT), Chiang Mai, 2018, pp. 1-2, doi: 10.1109/IWAIT.2018.8369795.

[9] A. Titoriya and S. Sachdeva, "Breast Cancer Histopathology Image Classification using AlexNet," 2019 4th International Conference on Information Systems and Computer Networks (ISCON), Mathura, India, 2019, pp. 708-712, doi: 10.1109/ISCON47742.2019.9036160.

[10] P. T. Nguyen, T. T. Nguyen, N. C. Nguyen and T. T. Le, "Multiclass Breast Cancer Classification Using Convolutional Neural Network," 2019 International Symposium on Electrical and Electronics Engineering (ISEE), Ho Chi Minh, Vietnam, 2019, pp. 130-134, doi: 10.1109/ISEE2.2019.8920916.

[11] M. Y. Hassan. Re: Why Deep Learning method perform better than other traditional Machine Learning methods?, International Islamic University Malaysia , Malaysia, 2016, Retrieved from: https://www.researchgate.net/post/Why-Deep-Learningmethod-perform-better-than-other-traditional-MachineLearningmethods/57fe15bf217e201ded3b07c1/citation/download. [12] K. O'Sheal and R. Nash, "An Introduction to Convolutional Neural Networks," 2015 Retrieved from: https://arxiv.org/abs/1511.08458. 